\title{
Inquérito Epidemiológico sobre tracoma em escolas pertencentes a IV Gerência Regional de Saúde do Estado de Pernambuco
}

\section{Epidemiological survey about trachoma in schools belonging to the IV Gerência Regional de Saúde located in the state of Pernambuco}

\author{
Josefa Elaine Silva Germinio \\ Mestranda pelo Programa de Pós-graduação em Engenharia Biomédica da Universidade Federal de Pernambuco. E- \\ mail: elainegerminio@ hotmail.com \\ Efraim Naftali Lopes Soares \\ Biomédico, Mestrando pelo Programa de Pós-graduação em ciência animal tropical. E-mail: efraimnaftali@gmail.com \\ Lucas Portela Silva \\ Mestrando pelo Programa de Pós-graduação em Medicina Tropical pela Universidade Federal de Pernambuco. E-mail: \\ portella.lucas.lp@gmail.com \\ Laura Emanuelle Silva \\ Bacharel em Biomedicina- Faculdades ASCES. E-mail: lauraemanuelle15@outlook.com \\ Ednaldo Ribeiro da Penha \\ Biólogo licenciado, Coordenador do programa de Tracoma da - IV Gerência Regional de Saúde. E-mail:
} tanairon@gmail.com

\begin{abstract}
Resumo: O tracoma é uma doença inflamatória ocular crônica que atinge a conjuntiva e a córnea, tendo como agente etiológico a Chlamydia trachomatis que provoca um quadro clinico de conjuntivite crônica, acompanhada de poucos sinais e sintomas, sendo apenas, prurido ocular, hiperemia leve, pouca ou nenhuma secreção ocular, sendo o diagnóstico do Tracoma estrategicamente clínico, e geralmente feito através do exame oftalmológico externo. O tratamento sistêmico é seletivo com antibiótico de ação sistêmica por via oral. O objetivo do estudo foi identificar possíveis infecções oculares em estudantes para se obter um perfil de prevalência. Foi realizado um estudo transversal com alunos do ensino fundamental, matriculados nas escolas da rede pública de alguns municípios pertencentes as quatro microrregiões da IV Gerência Regional de Saúde (GERES) considerados endêmicos para tracoma e também em alguns municípios não endêmicos que se dispuseram a participar do estudo. Todas as formas clínicas diagnosticadas nos estudantes examinados foram de TF- Tracoma Inflamatório Folicular. Foram examinados 25.594 estudantes de municípios prioritários e destes foram notificados e confirmados 541 casos do agravo. Quanto aos municípios não prioritários, foram realizados apenas 2.844 analises de infecções por tracoma em estudantes e apenas 83 casos positivos foram diagnosticados. A partir deste estudo foi possível concluir que algumas regiões da IV GERES apresentaram um índice de manifestação do tracoma que apesar de leve, mostra que a infecção ainda não está extinta, como preconiza o governo que aconteça em todo o Brasil até 2020. A região com maior prevalência de manifestação de tracoma foi a microrregião IX com cerca de 15,2\% do total de casos positivos, e durante os anos estudados, 2012 foi o que apresentou maior número de casos.
\end{abstract}

Palavras-chave: Chlamydia trachomatis, Infecção ocular, Inflamação folicular.

Abstract: Trachoma is a chronic inflammatory eye disease that affects the conjunctive and the cornea areas of the eyes, Chlamydia trachomatis causes a clinical effect of chronic conjunctivitis, accompanied by few signs and symptoms such as only, ocular itching, slight hyperemia, little or no eye discharge. Trachoma diagnosis is strategically clinical, and generally made through the external eye examination. Systemic treatment is selective with systemic acting antibiotic orally. The objective of this study was to identify eyes infections in students, to obtain the prevalence profile. A crosssectional study was done with elementary school students, enrolled in the public schools of some cities belonging the four micro-regions of the IV Gerencia Regional de Saude (GERES) considered endemic for trachoma and also, in some cities non-endemic which agreed to trace their infection profile. All clinical forms diagnosed in students examined were TF- Follicular inflammatory trachoma. 25,594 schoolchildren in priority municipalities were examined. From this total, 541 were reported and confirmed cases of the disorder. From non-priority municipalities, only 2,844 were performed trachoma infection in students and just 83 positive cases were diagnosed. From this study, it was possible to conclude that some regions of the IV GERES presented a low manifestation of trachoma, but it had been not extinguished yet, as recommended by the government that happens throughout Brazil by 2020. The regions which showed the highest prevalence of the infection was the micro-region IX, about $15,2 \%$ in a total of positive cases, and during the years studied, 2012 had the largest number of cases.

Keywords: Chlamydia trachomatis, Eye Infection, follicular inflammation. 


\section{INTRODUÇÃO}

O tracoma é uma doença inflamatória ocular crônica que atinge a conjuntiva e a córnea, tendo como agente etiológico a Chlamydia trachomatis uma bactéria Gram negativa de vida intracelular de sorotipos associados $\mathrm{A}, \mathrm{B}, \mathrm{Ba}$ e $\mathrm{C}$ e com tropismo pelas células epiteliais. Quando a reinfecção acontece inúmeras vezes, pode resultar em cicatrizes na conjuntiva palpebral, levando à formação de triquíase, estas lesões são fatores de risco para o aparecimento de alterações corneanas que induzem a incapacidade visual, estudos apontam o tracoma como a principal causa de cegueira evitável no mundo (LOPES et al, 2013).

No Brasil, o tracoma foi diagnosticado inicialmente no Nordeste a partir do século XVIII, com a deportação dos ciganos que haviam sido expulsos de Portugal e se estabeleceram nos estados do Ceará e Maranhão, constituindo o primeiro foco de tracoma no país (BARROS; LUNA; MEDINA, 2001). A prevalência do tracoma está diretamente relacionada ao baixo índice socioeconômico e à falta de saneamento básico, esta infecção é considerada pelas organizações internacionais da saúde como uma das doenças classificadas como negligenciadas, isso mostra que tem havido pouco ou nenhum investimento da indústria, no desenvolvimento de novos métodos de diagnóstico, fármacos e vacinas (SCHELLINI; SOUSA, 2012).

Em 2005, um mapa da distribuição global dos casos de tracoma foi realizado por Polack et al. através de levantamento de dados mostrando claramente que o Brasil é um país tem prevalência bastante significativa no número de casos de tracoma em toda a América (POLACK; BROOKER; KUPER, 2005).

A infecção pela Chlamydia trachomatis provoca um quadro clinico de conjuntivite crônica, acompanhada de poucos sinais e sintomas, sendo apenas, prurido ocular, hiperemia leve, pouca ou nenhuma secreção ocular (SCHELLINI; SOUSA, 2012). O período de incubação dura cerca de 5 a 12 dias e qualquer pessoa pode ser suscetível a contrair a infecção, sendo crianças as mais sensíveis, inclusive às reinfecções (BARROS; LUNA; MEDINA, 2001)

A principal forma de transmissão do tracoma é a direta, de olho a olho, mas também pode ocorrer de forma indireta, através de objetos pessoais contaminados. Alguns insetos, como a mosca doméstica e/ou a lambe-olhos podem atuar como vetores mecânicos (BARROS; LUNA; MEDINA, 2001).

A Organização Mundial de Saúde (OMS) espera que seja possível a erradicação definitiva do tracoma até o ano de 2020, tendo como principal ferramenta de combate o programa chamado "SAFE 2020", que visa manter o controle sobre à transmissão do tracoma e diminuição de suas consequências (DAMASCENO et al, 2009). SAFE é baseada em medidas simples e que envolvem principalmente higiene pessoal, melhores condições de vida e de saneamento $\mathrm{S}=$ cirurgia corretiva de triquíase; $\mathrm{A}=$ antibióticos; $\mathrm{F}=$ limpeza da face; $\mathrm{E}=$ saneamento (SCHELLINI; SOUSA, 2012).

O diagnóstico do Tracoma é estrategicamente clínico, e geralmente feito através do exame oftalmológico externo, devendo-se utilizar uma lupa do tipo binocular de 2,5 vezes de aumento. Durante o exame clinico é importante observar as pálpebras e a córnea, verificandose a presença ou ausência de entrópio, triquíase e opacificações corneanas. Em seguida, deve-se everter a pálpebra superior e examinar a área central da conjuntiva tarsal. Pode-se observar dois tipos de reação conjuntival na inflamação tracomatosa: os folículos e a infiltração difusa, que podem ocorrer simultaneamente. $\mathrm{O}$ diagnóstico clínico do Tracoma baseia-se na verificação da presença ou ausência de cinco sinais-chave: Inflamação Tracomatosa Folicular (TF), Inflamação Tracomatosa Intensa (TI), Cicatrização Conjuntival Tracomatosa (TS), Triquíase Tracomatosa (TT) e Opacificação Corneana (CO) segundo o manual de controle do tracoma (BARROS; LUNA; MEDINA, 2001). A confirmação laboratorial através do exame de cultura microbiológica é utilizada, para contatar a presença do agente etiológico, e não para a confirmação de cada caso (SCHELLINI; SOUSA, 2012).

O tratamento sistêmico é seletivo com antibiótico de ação sistêmica por via oral, indicado para pacientes com Tracoma intenso (TI) ou casos de TF ou TI que não respondam bem ao medicamento tópico. $\mathrm{O}$ mais comum e recomendado pelo Manual de controle do Tracoma é Eritromicina $250 \mathrm{mg}$ quatro vezes ao dia, durante três semanas e deve ser usado com critério e acompanhamento médico devido às possíveis reações adversas.

De acordo com a definição da Secretaria Estadual de Saúde (SES) as GERES tem a missão de gerenciar a política regional de saúde a partir do conhecimento do município, do território e da região. $O$ Estado de Pernambuco é dividido em 12 Gerências Regionais de Saúde (GERES) com intensão de regular, monitorar e avaliar as metas pactuadas por cada município abrangente. Outra atribuição é coordenar a construção da rede de atenção regional, garantindo o acesso da população aos serviços de saúde. A IV Regional localizada no agreste do Estado de Pernambuco é a responsável por monitorar 32 municípios, e sendo a maior gerencia regional é também dividida em quatro microrregiões.

A subdivisão da IV GERES envolve a microrregião VI contando com os municípios de Caruaru, Riacho das Almas, São Caetano, Altinho, Agrestina, Ibirajuba, Cupira, Panelas e Jurema. A microrregião VII, da qual fazem parte os municípios de Bezerros, Gravatá, Saíre, Camocim de São Félix, São Joaquim do Monte, Bonito, Barra de Guabiraba e a microrregião VIII, com os municípios de Belo Jardim, Tacaimbó, Sanharó, Poção, Pesqueira, Alagoinha, São Bento do Uma e cachoeirinha. Por fim, a microrregião IX inclui os municípios de Santa Cruz do Capibaribe, Taquaritinga do Norte, Vertentes, Toritama, Brejo da Madre de Deus, Jataúba, Frei Miguelinho e Santa Maria do Cambucá. Dos municípios citados nove são considerados como prioritários no monitoramento de Tracoma, e a IV Gerência Regional é o órgão responsável por levantar os dados epidemiológicos sobre a situação de cada um destes municípios sejam prioritários ou não.

O Tracoma não é uma doença de notificação compulsória, no entanto é importante e recomendável que sejam feitos registros sistemáticos dos dados mínimos 
sobre os casos detectados e tratados. Este trabalho tem como objetivo identificar possíveis infecções oculares em estudantes entre faixa etária vulnerável a adquirir infecções no ambiente escolar, na tentativa de se obter um perfil de prevalência destas infecções diagnosticadas e trata-las.

\section{MATERIAL E MÉTODOS}

Foi realizado um estudo de prevalência com alunos do ensino fundamental, matriculados nas escolas da rede pública de alguns municípios pertencentes as quatro microrregiões da IV Gerência Regional de Saúde (GERES) considerados endêmicos para tracoma e também em alguns municípios não endêmicos que se dispuseram a traçar o seu perfil de infecção.

Entre os municípios participantes do estudo podemos citar: Agrestina, Barra de Guabiraba, Bonito, Cupira, Camocim de São Félix, Frei Miguelinho, Iburajuba, Jataúba, Jurema, Panelas, Pesqueira, Poção, Santa Maria do Cambucá, São Joaquim do Monte e Vertentes todos localizados no interior do Agreste Pernambucano (Figura 1).

Figura 1. Mapa dos municípios pertencentes a IV Regional de saúde de Pernambuco, separados por microrregião.

\section{REGIĀO DE SAÚDE/CARUARU}

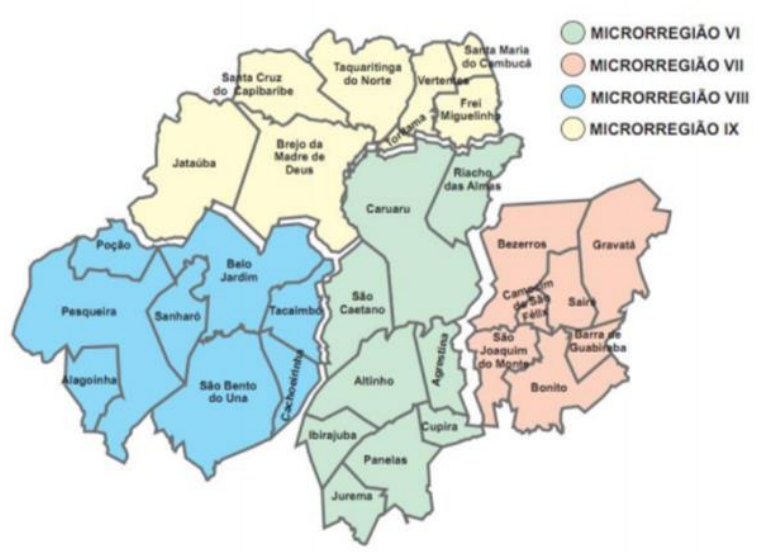

Fonte: IV GERES/PE (2015)

Foram examinadas um total de 28.438 crianças entre a faixa etária de 5 a 14 anos, sendo 25.594 estudantes de municípios prioritários e 2.844 de municípios não prioritários devidamente matriculados em escolas públicas. Participaram da realização dos exames, técnicos da secretaria estadual de saúde treinados pelo Ministério da Saúde, enfermeiros, Agentes Comunitários de Saúde e técnicos da epidemiologia municipal.

Para o exame clínico foi utilizado uma lupa de 2,5 vezes de magnitude sob iluminação com lanternas ou natural, tendo como objetivo avaliar as alterações presentes nos cílios, pálpebras das conjuntivas e córneas em ambos os olhos dos escolares matriculados nas escolas municipais, como preconiza o protocolo do Ministério da Saúde. Os alunos que apresentaram resultado positivo após o exame, foram tratados e acompanhados a cada seis meses no primeiro e segundo contexto, como preconiza o formulário padrão de controle de casos.
A IV Gerência Regional de Saúde localizada na cidade de Caruaru coordena a rede de atenção regional, monitorando e garantindo o acesso da população aos Serviços de saúde aos 1.324.382 habitantes distribuídos em quatro microrregiões VI, VII, VIII, IX. Cumprindo as exigências citadas, entre os anos de 2009 a 2014 foram realizadas ações de monitoramento de infecção ocular em diversos municípios das microrregiões.

\section{RESULTADOS E DISCUSSÃO}

$\mathrm{O}$ estudo realizado indicou que a maior prevalência de casos de infecção ocular foi no ano de 2012, representando 63,09\%. Nos anos de 2011 a mostra representou $11,89 \%$ e em 2013 os casos notificados chegaram a 13,60\%. Em 2009 e em 2010, os casos notificados não chegaram a $(8 \%)$. Quanto aos municípios não prioritários, foram realizados apenas 2.844 analises de infecções por tracoma em estudantes e apenas 83 casos positivos foram diagnosticados. A faixa etária acometida foram de escolares entre 5 a 14 anos.

Os resultados do inquérito realizados nos municípios pertencente as IV GERES foram também divididos a partir das microrregiões, para que fosse possível obter o perfil de infecção por tracoma em cada uma. Porém não há resultados de todos os municípios pois vários destes, principalmente os classificados como não prioritários, não foram incluídos na campanha realizada pela IV GERES.

A microrregião VI conta como os municípios de Caruaru, Riacho das Almas, São Caetano, Altinho, Agrestina, Ibirajuba, Cupira, Panelas e Jurema. Apenas obteve-se resultados de cinco municípios dos quais foram examinados num total 8,988 escolares $(12.1 \%$ de casos positivos). Observa-se que todos os municípios apresentaram um percentual abaixo do valor considerado aceitável de 5\%, mas os considerados não prioritários apresentaram um percentual mais alto em relação aos prioritários (Figura 2).

A microrregião VII, da qual fazem parte os municípios de Bezerros, Gravatá, Saíre, Camocim de São Félix, São Joaquim do Monte, Bonito e Barra de Guabiraba o inquérito foi realizado apenas em quatro municípios totalizando 7.729 (12\% casos positivos) análises, todos com percentual abaixo da referência desejável, e dos resultados obtidos observa-se que Ibirajuba e Panelas apesar de considerados não prioritários foram os municípios com maior percentual de casos de infecção por Chlamydia trachomatis (Figura 3)

Da microrregião VIII, fazem parte os municípios de Belo Jardim, Tacaimbó, Sanharó, Poção, Pesqueira, Alagoinha, São Bento do Uma e Cachoeirinha. Apenas em dois municípios, os escolares foram examinados num total de 7.654 (4,9\% casos positivos). Pesqueira e Poção são ambos considerados prioritários e apesar de abaixo do percentual considerado aceitável, obtiveram percentuais diferentes. (Figura 4)

Por fim, a microrregião IX inclui os municípios de Santa Cruz do Capibaribe, Taquaritinga do Norte, Vertentes, Toritama, Brejo da Madre de Deus, Jataúba, Frei Miguelinho e Santa Maria do Cambucá. Quatro municípios tiveram escolares examinados totalizando 3.995 (15,2\% casos positivos). Apenas Vertentes é considerado prioritário e apesar disso, foi o que obteve 
menor percentual de escolares diagnósticos com tracoma. examinados obteve um percentual elevado de infecção por Nesta microrregião destaca-se o município de Frei tracoma sendo de 7.9\% superior ao desejável (Figura 5). Miguelinho que apesar do baixo quantitativo de escolares

Figura 2 - Total de pacientes examinados, casos positivos e incidência em cada município prioritário e não prioritário da microrregião VI.

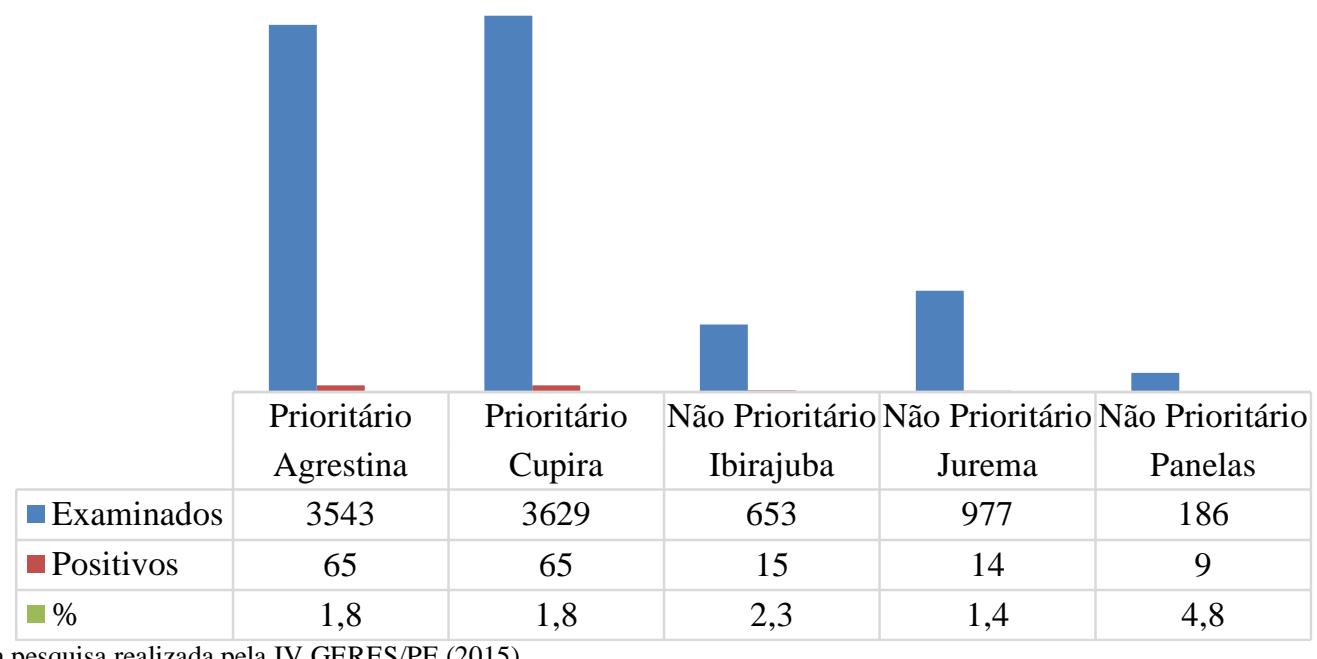

Fonte: Dados da pesquisa realizada pela IV GERES/PE (2015).

Figura 3- Total de pacientes examinados, casos positivos e incidência em cada município prioritário e não prioritário da microrregião VII.

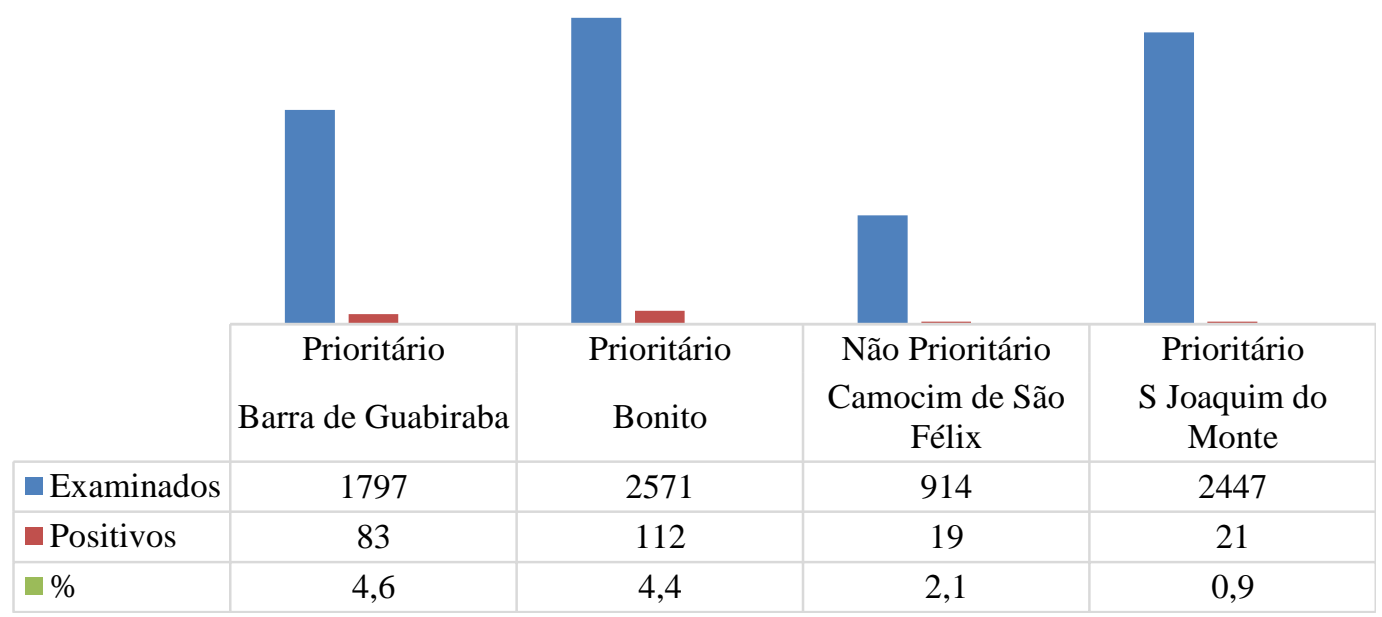

Fonte: Dados da pesquisa realizada pela IV GERES/PE (2015).

Figura 4 - Total de pacientes examinados, casos positivos e incidência em cada município prioritário e não prioritário da microrregião VIII.

\begin{tabular}{|c|c|c|}
\hline & $\begin{array}{l}\text { Prioritário } \\
\text { Pesqueira }\end{array}$ & $\begin{array}{c}\text { Prioritário } \\
\text { Poção }\end{array}$ \\
\hline Examinados & 5458 & 2196 \\
\hline Positivos & 59 & 83 \\
\hline$\%$ & 1,1 & 3,8 \\
\hline
\end{tabular}

Fonte: Dados da pesquisa realizada pela IV GERES/PE (2015).

Rev. Bra. Edu. Saúde v. 6, n.1, p. 22-27, 2016 
Figura 5 - Total de pacientes examinados, casos positivos e incidência em cada município prioritário e não prioritário da microrregião IX

\begin{tabular}{|c|c|c|c|c|}
\hline & $\begin{array}{l}\text { Não Prioritário } \\
\text { Frei Miguelinho }\end{array}$ & $\begin{array}{l}\text { Não Prioritário } \\
\text { Jataúba }\end{array}$ & $\begin{array}{c}\text { Não Prioritário } \\
\text { Santa } \mathrm{M}^{\mathrm{a}} \text { Cambuca }\end{array}$ & $\begin{array}{l}\text { Prioritário } \\
\text { Vertentes }\end{array}$ \\
\hline Examinados & 254 & 705 & 60 & 2976 \\
\hline Positivos & 20 & 19 & 2 & 39 \\
\hline$\%$ & 7,9 & 2,7 & 3,3 & 1,3 \\
\hline
\end{tabular}

Fonte: Dados da pesquisa realizada pela IV GERES/PE (2015).

É importante citar que todas as formas clínicas diagnosticadas nos estudantes examinados foram de Tracoma Inflamatório Folicular, descartando qualquer outra forma infecciosa mais grave como: Inflamação Tracomatosa Intensa, Cicatrização Conjuntival Tracomatosa, Triquíase Tracomatosa, e Opacificação Corneana descritas no manual de controle do tracoma (BARROS; LUNA; MEDINA, 2001). Todos os estudantes diagnosticados com a infecção, foram encaminhados para a unidade de saúde responsável para realização do tratamento.

Em média quatro municípios de cada microrregião foram monitorados, o maior índice de infecção foi na microrregião IX, com cerca de $15,2 \%$ do total de casos diagnosticados em escolares entre as faixas etárias de 5 a 14 anos. Apesar de apenas um município ter o percentual acima do valor aceitável, sendo este Frei Miguelinho apresentando 7,9, \% nota-se que a infecção por Chlamydia trachomatis ainda está presente em diversos municípios da IV Gerencia Regional de Saúde. Observa-se também que em algumas das microrregiões citadas os municípios considerados não prioritários apresentaram um percentual um pouco mais elevado, comparados a alguns considerados como prioritários para o tratamento de tracoma.

Alguns estudos de prevalência realizados em diferentes regiões do Brasil tiveram os mais diversos resultados, porém todos mostram que esta infecção está longe de ser considerada erradicada no país. Alguns estados apresentaram baixos índices de infecção tais como: Rio Grande do Norte (3,61\%), Bahia (3,58\%), Paraíba (3,81\%), Roraima (4,34\%) e São Paulo $(4,11 \%)$. Outros estados, como Rio Grande do Sul (4,60\%), Tocantins $(5,33 \%)$, Sergipe $(5,84 \%)$, Paraná $(6,2 \%)$, Espírito Santo $(4,74 \%)$, Ceará $(7,81 \%)$ e Acre $(8,34 \%)$ apresentam índices de prevalência mais elevados de infecção ocular por Chlamydia trachomatis (BARROS; LUNA; MEDINA, 2001).

A taxa de detecção de tracoma encontrada nos municípios da IV GERES são baixos comparados aos outros estudos ao redor do país, mas para que seja possível erradicar a infecção muitas estratégias de atenção à saúde devem sem implantadas em todo território, destacando-se a melhoria das condições sanitárias da população.

\section{CONCLUSÕES}

A partir deste estudo foi possível concluir que algumas regiões da IV Gerência Regional de Saúde apresentaram um índice de manifestação do tracoma que apesar de leve, mostra que a infecção ainda não está extinta, como preconiza o governo que aconteça em todo o Brasil até 2020, ressaltando-se a importância de educar a população jovem através de aulas educativas, noções de higiene e de percepção quanto aos sinais e sintomas.

A região com maior prevalência de manifestação de tracoma foi a microrregião IX com cerca de 15,2\% do total de casos positivos. Durante os anos pesquisados, 2012 apresentou maior índice de infecção por casos confirmados.

\section{REFERÊNCIAS BIBLIOGRÁFICAS}

BAILEY, R.; LIETMAN, T. The SAFE strategy for the elimination of trachoma by 2020: will it work? Bull World Health Organ, v.79,n.3, p.233-236, 2007.

BARROS, O. M.; LUNA, E. A.; MEDINA, N. H. et al. Manual de controle do tracoma. Brasília: Ministério da Saúde, Fundação Nacional da Saúde; 2001.

DAMASCENO, R. W.; SANTOS, R. R.; CAVALCANTI, T. R, et al. Tracoma: estudo epidemiológico de escolares em Alagoas - Brasil. Arq. Bras. Oftalmol. Maceió- AL, v. 72, n.3, p. 355-359, 2009.

FERRAZ, L. C. B.; SCHELLINI, S. A.; PADOVANI, C. R. et al. Tracoma em crianças do ensino fundamental no município de Bauru Estado de São Paulo, Brasil. Arq. Bras. Oftalmol. São Paulo, v. 73, n. 5, p. 433-437, 2010.

KOIZUMI, J. K.; MEDINA, N. H.; D'AMARAL, R. K. K. et al. Prevalência de tracoma em pré-escolares e escolares no município de São Paulo. Rev. Saúde Pública, São Paulo, v. 39, n. 6, p. 937-942, 2005.

LOPES, M. F. C.; LUNA, E. J. A.; MEDINA, N. H. et al. Prevalência de tracoma entre escolares brasileiros. Rev. Saúde Pública, São Paulo, v. 47, n. 3, p. 451-459, 2013. 
MORSCHIBACHER, R.; MEDINA, N.H; LUNA, E. J. A. et al. Estudo epidemiológico de tracoma. Arq. Bras. Oftalmol. São Paulo, v.74, n. 3, p.227-229, 2011.

POLACK, S.; BROOKER, S.; KUPER, H. et al. Mapping the global distribution of Trachoma. Bulletin The World Health Organization, v.83, n.12, p. 913-919, 2005.

SCHELLINI, S. A.; SOUSA, R. L. F. Tracoma: ainda uma importante causa de cegueira. Rev. Bras. Oftalmol. São Paulo, v.71, n. 3, p.199-204, 2012.

SEADL, C. F.; ORAVEC, R.; POSER, B. V. et al. Diagnóstico laboratorial da infecção pela Clamydia trachomatis: vantagens e desvantagens das técnicas. Jornal Brasileiro de Patologia e Medicina Laboratorial. Rio de Janeiro, v. 38, n. 2, p. 125-133, 2002. 